\title{
Sigmoid sinus thrombosis in an elderly client with chronic varicose vein in the leg: a case report
}

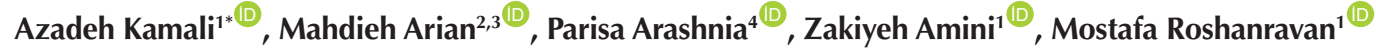 \\ 'Department of Nursing, Faculty of Nursing and Midwifery, North Khorasan University of Medical Sciences, Bojnurd, IR Iran. \\ ${ }^{2}$ Department of Nursing, School of Nursing and Midwifery, Semnan University of Medical Sciences, Semnan, Iran. \\ ${ }^{3}$ Student Research Committee, Semnan University of Medical Sciences, Semnan, Iran. \\ ${ }^{4}$ Infectious Disease Specialist, North Khorasan University of Medical Sciences, Bojnurd, Iran.
}

*Corresponding Author: Azadeh Kamali, Tel: +98-5832296909, Email: a.kamali@nkums.ac.ir

\begin{abstract}
Dehydration and malignancies are known as the most frequent predisposing factors of cerebral venous sinus thrombosis in older adults despite the fact that $30 \%$ of them remain idiopathic cerebral venous thrombosis (CVT) cases with no clear etiology. Cases of left sigmoid sinus involvement were rarely reported. Herein, an elderly case of sigmoid sinus thrombosis with unilateral hearing loss with chronic varicose vein in the leg was reported. Sigmoid sinus thrombosis that affects hearing is one of the rare complications of otitis media. Whether or not a large chronic varicose vein in the leg can serve as a predisposing factor for CVT requires further studies. Successful management of anticoagulant therapy prevented serious complications in the patient.

Keywords: Sigmoid sinus thrombosis, Cerebral sinus thrombosis, Unilateral hearing loss, Elderly patients, Varicose vein
\end{abstract}

Received: 28 October 2019, Accepted: 8 January 2020, ePublished: 29 April 2020

Introduction

Cerebral venous sinus thrombosis is defined as the formation of a clot in the dural venous sinuses which drain both the brain's superficial veins and deep venous system (1). This condition can occur at any age, however, the highest incidence rates have been reported in women of reproductive age, young adults, and children (2-4). Predisposing factors for cerebral venous thrombosis (CVT) include the use of contraceptives, pregnancy, and parturition. In older adults, however, such factors are very rare and dehydration and malignancies are the most frequent predisposing factors (5). Moreover, 30\% of the CVT cases in older adults are idiopathic with no clear etiology (6). Although hormonal changes, trauma, infections, hematologic diseases, anemia, and noninfectious inflammatory diseases, such as Behcet's disease and inflammatory bowel disease, are the main risk factors for CVT, no CVT risk factors can be found in $17 \%$ to $27 \%$ of the adult patients and $2 \%$ of pediatric cases $(7,8)$. In young patients, headache is the first symptom followed by vision changes such as blurred and double vision, vomiting, leg edema, seizure, changes in the level of consciousness, and dizziness. Among the elderly, headache is less frequent, whereas reduced consciousness and mental status changes are more frequent $(5,9)$.
The statistics regarding the frequency of the site of CVT vary. Many studies have reported the involvement of sagittal and lateral sinuses in $70 \%$ and $60 \%$ to $70 \%$ of cases, respectively. Such cases do not occur independently and are often caused by otitis media (7). A study in Iran indicated that left sigmoid sinus involvement was less frequent than the involvement of the right side $(6.7 \%$ vs. $16.7 \%$ ) and most cases of involvement were in the right cerebral hemisphere (10). Another study in the northeast of Iran reported the frequency of concurrent involvement of upper sagittal and lateral sinuses as 65\%. Sigmoid sinus involvement was found in $5 \%$ of the cases (6). Some studies have reported a higher prevalence of transverse sinus thrombosis $(70 \%)$ than sigmoid sinus thrombosis $(53 \%)(11,12)$. Sigmoid sinus thrombosis is a serious but rare complication following otitis media (13).

\section{Case Presentation}

A 68-year-old man from one of the villages around the city of Bojnurd, North Khorasan province presenting with dizziness and nausea was referred to the hospital by a neurologist. He emphasized the loss of hearing in the left ear. According to the history obtained from his wife, his dizziness, nausea, and loss of hearing in the left ear had severely and suddenly started in the street on September

(C) 2020 The Author(s); Published by Shahrekord University of Medical Sciences. This is an open-access article distributed under the terms of the Creative Commons Attribution License (http://creativecommons.org/licenses/by/4.0), which permits unrestricted use, distribution, and reproduction in any medium, provided the original work is properly cited. 
10, 2017 and prevented him from standing or walking. The patient was examined immediately by an ear, nose, and throat specialist and diagnostic magnetic resonance imaging (MRI) was requested. The results suggested the likelihood of left jugular vein thrombosis. However, with the symptoms abating following this diagnosis, the patient refused to pursue treatment procedures in spite of physicians' advice. Four months later (February 4, 2018), dizziness started again. The patient visited a neurologist and an MRI was obtained. The results suggested left sigmoid sinus thrombosis and the patients was admitted to Imam Hassan Hospital. Magnetic resonance venography confirmed the diagnosis (Figure 1). Audiometry indicated moderate unilateral sensorineural deafness. Vital signs, chest X-ray, and abdominal and pelvic ultrasound were normal. Prostate-specific antigen, ferritin, peripheral blood smear, vitamin B12 tests, and stool exam were carried out which yielded normal results. The patient had no fever or increased white blood cell count. No oral or dental infections were present. He was not addicted to any opioids. His platelet count was 130000 and blood sugar level was in the normal range. He had cataract surgery three years ago. He did not report fever or headache in the past few days and had no ear infection or head trauma. He did not report the use of a particular medication, drug addiction, or other diseases. He had no gastrointestinal inflammatory diseases, constipation, or diarrhea. No specific reason was found for the thrombosis during the few days of his hospital stay. During hospitalization, the patient was on bed rest and his wife provided the history.

Heparin infusion (1000 unit/hour) was done using a heparin pump. Constant monitoring of International Normalized Ratio (INR) and partial thromboplastin time/prothrombin time (PTT/PT) tests were carried out. Additionally, treatment with oral anticoagulants continued. Levetiracetam $500 \mathrm{mg}(1 / 2)$ BID, acetazolamide

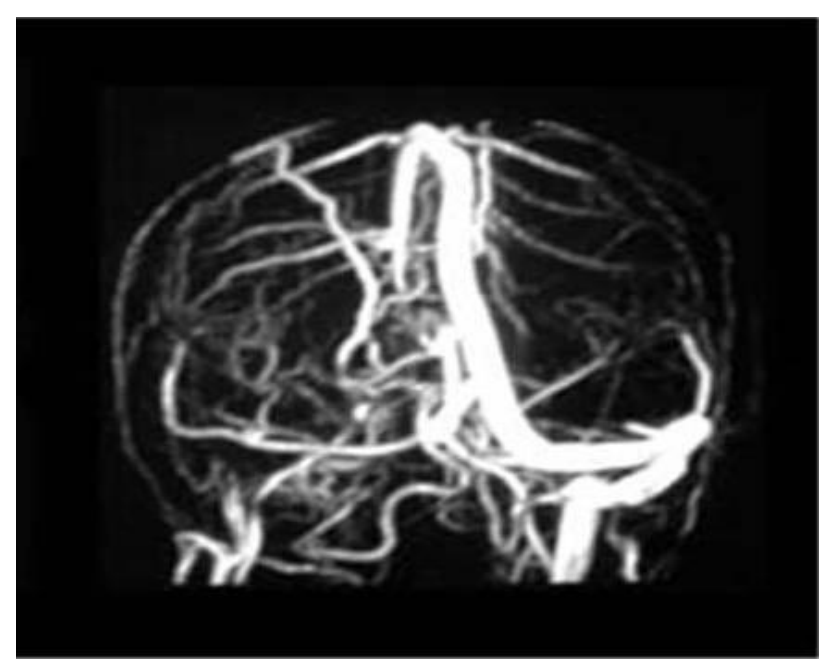

Figure 1. Magnetic resonance venography of the brain showing thrombosis in the left sigmoid sinus.
$250 \mathrm{mg} / \mathrm{d}$, warfarin $5 \mathrm{mg} / \mathrm{d}$, and Amp ranitidine $50 \mathrm{mg}$ TDS (three times per day) were administered. A few days before discharge, the patient was assigned to a native nursing student in the evening shift. The development of trust led to proper communication with the patient's wife and disclosure of symptoms which had not been previously discussed by a female therapist due to embarrassment, ignorance, or religious and cultural issues. His wife reported a chronic varicose vein in the patient's left leg that had frequently caused wounds. Although she reported that the recent wound had existed for several months, he did not seek any medical treatment and only used homemade remedies. After gaining the patient's trust and informing the physician (while observing the privacy of the patient), the examination was carried out and large bulging veins in the left leg and signs of a dry wound $(5 \times 5 \mathrm{~cm})$ with no discharge were observed on the inner shin (Figure 2). The patient had hidden the wound either under the blanket or by wearing socks. He had not used any specific treatments (e.g., bandage or wearing special socks) except for resting and raising the leg to reduce leg edema. Skin examinations, i.e., wound assessment and malignancy evaluation, were requested immediately. The results showed no malignancy and the wound was merely an old healed ulcer caused by varicose veins. Doppler ultrasound of the left lower limbs (venous and arterial) was carried out and no deep vein thrombosis (DVT) was reported. Heparin infusion was continued and the patient was discharged with an INR of 2. An oral anticoagulant (warfarin) was prescribed for him. At the eight-month follow-up, decreased hearing was still present in the left ear, but ulcers caused by varicose veins fully healed with only a little itching remaining. The use of Warfarin and INR monitoring were continued.

\section{Discussion}

Sensorineural hearing loss is an ontological emergency that

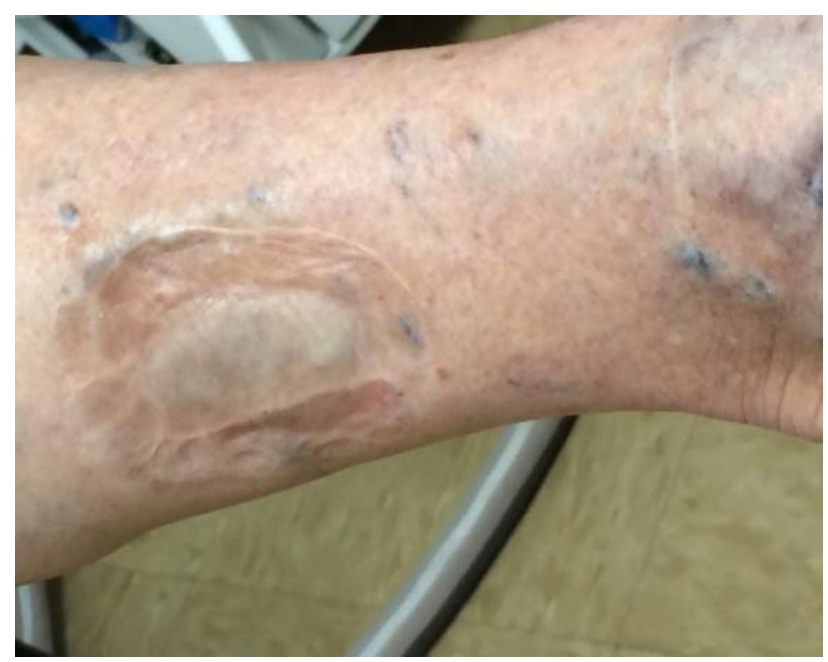

Figure 2. Large bulging veins in the left leg and signs of a dry wound on the inner shin. 
requires immediate assessment to find possible etiology that may be life-threatening. However, $85 \%$ to $90 \%$ of such cases are idiopathic and have no known cause (14). CVT is a kind of venous thromboembolism responsible for $0.5 \%$ to $3 \%$ of stroke cases. While CVT is more prevalent in young people, cerebral arterial strokes mostly affect older adults (15). Lateral cerebral sinuses, i.e. transverse and sigmoid sinuses, are present in the blood drains from the cerebellum, brain stem, and the posterior portions of brain hemispheres (16). According to Virchow's triad, the risk factors for venous thrombosis include venous stasis, changes in prothrombotic state, and blood vessel (veins/sinuses) damage. Therefore, any factor exacerbating each of these three factors can be considered as a risk factor (17). CVT is a multifactorial disease and finding one of these factors should not prevent pursuing other possible factors because ignoring any of these factors can lead to the recurrence of the disease (18). The main cause of thrombosis is not diagnosed in $30 \%$ of the cases. In our patient, the cause(s) of sigmoid sinus thrombosis could not be detected. Since no evidence of fever and infection, malignancy, impaired coagulation, or trauma was found, the likelihood of an association between sigmoid sinus thrombosis and the complications resulting from extensive varicose veins of the leg was suspected and discussed. Ulcers caused by varicose veins of the lower limbs often occur above the ankle and are more common in older adults and women. The prevalence of such ulcers increases with age to reach 20 in 1000 people aged over 80 years old (19). The impairment in leg veins and the consequent complications of ulcers caused by varicose veins might contribute to the incidence of CVT. However, ultrasound and laboratory tests showed no underlying impairment in this regard (14). CVT is a multifactorial disease with a diverse range of gender-related clinical symptoms. The condition has different causes in different countries (16). Sigmoid sinus thrombosis that affects hearing is one of the rare complications of otitis media (20). Based on Virchow's triad, changes in the wall of varicose veins of the leg are among factors causing DVT and its consequent complications (e.g., pulmonary thromboembolism). Whether or not a large chronic varicose vein in the leg can serve as a predisposing factor for CVT requires further studies.

\section{Conclusion}

Sigmoid sinus thrombosis that affects hearing is one of the rare complications of otitis media. Whether or not a large chronic varicose vein in the leg can serve as a predisposing factor for CVT requires further studies. Successful management of anticoagulant therapy prevented serious complications in the patient.

Conflict of Interest

None declared.
Ethical Approval

Informed consent was obtained the patient for publication of the report.

Authors' Contributions

AK and MA: Development of the original idea, Study concept and design, Acquisition of data, and manuscription

ZA and MR: Literature search, participate in abstract and drafting of manuscript

PA: Supervision of study and Critical revision

All Authors give final approval of the version to be submitted.

\section{References}

1. Ferro JM, Canhão P, Stam J, Bousser MG, Barinagarrementeria F. Prognosis of cerebral vein and dural sinus thrombosis: results of the International Study on Cerebral Vein and Dural Sinus Thrombosis (ISCVT). Stroke. 2004;35(3):664-70. doi: 10.1161/01.str.0000117571.76197.26.

2. Ropper AH, Brown RH. Adams and Victor's Principles of Neurology. 8th ed. New York: McGraw-Hill; 2005.

3. Ashjazadeh N, Borhani Haghighi A, Poursadeghfard M, Azin $\mathrm{H}$. Cerebral venous-sinus thrombosis: a case series analysis. Iran J Med Sci. 2011;36(3):178-82.

4. Martinelli I, Battaglioli T, Pedotti P, Cattaneo M, Mannucci PM. Hyperhomocysteinemia in cerebral vein thrombosis. Blood. 2003;102(4):1363-6. doi: 10.1182/blood-2003-02-0443.

5. Ferro JM, Canhão P, Bousser MG, Stam J, Barinagarrementeria F. Cerebral vein and dural sinus thrombosis in elderly patients. Stroke. 2005;36(9):1927-32. doi: 10.1161/01. str.0000177894.05495.54.

6. Nikkhah K, Sasannejad P, Azarpazhoh M, Saidi M, Afshar L. Brain venous thrombosis. Medical Journal of Mashhad University of Medical Sciences. 2009;52(3):147-50. doi: 10.22038/mjms.2009.5430. [Persian].

7. Bousser MG, Ferro JM. Cerebral venous thrombosis: an update. Lancet Neurol. 2007;6(2):162-70. doi: 10.1016/s14744422(07)70029-7.

8. Siddiqui FM, Kamal AK. Incidence and epidemiology of cerebral venous thrombosis. J Pak Med Assoc. 2006;56(11):485-7.

9. Farzadfard MT, Foroughipour M, Yazdani S, Ghabeli-Juibary A, Rezaeitalab F. Cerebral venous-sinus thrombosis: risk factors, clinical report, and outcome. A prospective study in the North East of Iran. Caspian J Neurol Sci. 2015;1(3):27-32.

10. Khomand P, Ahsan B, Gharibi F, Khadami AH. An evaluation of the frequency of cerebral venous sinus thrombosis and its associated factors in Sanandaj, 2010-2011, Iran. Qom University of Medical Sciences Journal. 2014;8(3):49-54. [Persian].

11. Coutinho JM, Zuurbier SM, Aramideh M, Stam J. The incidence of cerebral venous thrombosis: a cross-sectional study. Stroke. 2012;43(12):3375-7. doi: 10.1161/strokeaha.112.671453.

12. Idbaih A, Boukobza M, Crassard I, Porcher R, Bousser MG, Chabriat $\mathrm{H}$. MRI of clot in cerebral venous thrombosis: high diagnostic value of susceptibility-weighted images. Stroke. 2006;37(4):991-5. doi: 10.1161/01.STR.0000206282.85610. ae.

13. Arian M, Kamali A, Tabatabaeichehr M, Arashnia P. Septic cavernous sinus thrombosis: a case report. Iran Red Crescent Med J. 2016;18(8):e34961. doi: 10.5812/ircmj.34961.

14. Ishak MN, Nik-Abdul-Ghani NM, Mohamad I. Sudden bilateral sensorineural hearing loss secondary to cerebral venous thrombosis. Iran J Otorhinolaryngol. 2018;30(97):113-6.

15. Bousser MG, Crassard I. Cerebral venous thrombosis, pregnancy and oral contraceptives. Thromb Res. 2012;130 
Suppl 1:S19-22. doi: 10.1016/j.thromres.2012.08.264.

16. Alvis-Miranda HR, Milena Castellar-Leones S, Alcala-Cerra G, Rafael Moscote-Salazar L. Cerebral sinus venous thrombosis. J Neurosci Rural Pract. 2013;4(4):427-38. doi: 10.4103/09763147.120236.

17. Saposnik G, Barinagarrementeria F, Brown RD, Jr., Bushnell CD, Cucchiara B, Cushman M, et al. Diagnosis and management of cerebral venous thrombosis: a statement for healthcare professionals from the American Heart Association/American Stroke Association. Stroke. 2011;42(4):1158-92. doi: 10.1161/ STR.0b013e31820a8364.

18. Ruiz-Sandoval JL, Chiquete E, Bañuelos-Becerra LJ, Torres-
Anguiano C, González-Padilla C, Arauz A, et al. Cerebral venous thrombosis in a Mexican multicenter registry of acute cerebrovascular disease: the RENAMEVASC study. J Stroke Cerebrovasc Dis. 2012;21(5):395-400. doi: 10.1016/j. jstrokecerebrovasdis.2011.01.001.

19. Knott L. Venous Leg Ulcers. Availeble from: https://patient.info/ doctor/venous-leg-ulcers-pro.

20. Ropposch T, Nemetz U, Braun EM, Lackner A, Tomazic $\mathrm{PV}$, Walch C. Management of otogenic sigmoid sinus thrombosis. Otol Neurotol. 2011;32(7):1120-3. doi: 10.1097/ MAO.0b013e31822a1ec0. 